\title{
Model of bifurcation prediction and innovation sustainability of energy facilities
}

\author{
Mikhail Slesarev ${ }^{*}$ and Artur Kovrigin \\ Moscow State University of Civil Engineering, Yaroslavskoe shosse, 26, Moscow, 129337, Russia
}

\begin{abstract}
Innovative technological order is predicted, defined by "mechatronics" - an integrating structure for power engineering, mechanics, and artificial intelligence, which together with "green" technologies of production, accumulation, transformation and transmission of information, energy and materials, generate smart houses, smart cities, smart energy objects. A model is proposed for predicting bifurcations and innovative sustainability of energy facilities with an acceptable reliability of research provided by the mathematical apparatus of motion stability.
\end{abstract}

\section{Predicting a Green Technological Way}

In the last decade in Russia, the level of requirements for the complexity of experimental studies and surveys has significantly increased before the start of the construction of energy facilities [1], [2], [3]. Interstate and national standards for the management of experiment and research for construction appeared, market attributes of certification and risk insurance are used when conducting surveys for construction, modern methods of experimental research and testing, as well as measuring instruments are used [4], [5]. The legislation of Russia has been supplemented with legal and regulatory documents in the field of intellectual property protection, innovation and research, metrology and measurement uniformity.

Advanced developments in the field of information technologies and communications, as well as the rich experience of researchers in the strength properties of soils and rocks of bases, building materials, structures and building systems, require adaptation to the practical organization of the processes of experimental research and research in construction innovations [6], [7].

A simple reduction of all questions to the complexity in the production of research innovations may cause damage due to the loss of the ability to catch bifurcations in this process. Bifurcations create preconditions for both positive and negative synergistic effects during experimental studies, for example, studies of the soils and rocks of the foundations of large construction projects, which, as a rule, are energy facilities [8], [9], [10].

\footnotetext{
* Corresponding author: slesarev@mgsu.ru
} 


\section{2 "Green" Mechatronic Technologies}

Mechatronics [5] is a scientific technical and technological direction of a promising technological order in the development of the technosphere, integrating power engineering, mechanics, and artificial intelligence in conjunction with advanced technologies in the production, accumulation, transformation and transmission of information, energy and materials, dealing with various aspects of application and development smart devices, smart vehicles, smart houses, smart cities, smart energy objects, and finally a smart planet with global artificial intelligence ectom.

Mechatronic technology [5] is based on the practical possibility of creating a unified technology in which electronic devices, optical components, energy and mechanical structures of multicomplex devices with artificial intelligence are integrated into a single integrated system.

The energy of mechatronic technology is focused on hybrid [5], [8] technology of energy supply using combined methods and devices for the transfer, storage and conversion of energy across the whole gamut, including from nuclear reactors to molecular type generators using fine ceramics and composites with high-temperature superconductivity properties . A necessary property of mechatronic energy components is the possibility of embedding into the design mechatronic objects of any configuration and size, as well as the possibility of autonomous power generation or recharging from external energy sources in a non-contact-inductive manner and by converting other types of energy into electrical energy. Solar cells, thermal converters, strain gauges, energy converters of molecular and nuclear decay, etc., can be used as converters into electrical energy [13], [14], [15].

\section{Simulation and Experiment}

The most important areas of experimentation and modeling of the technosphere under the influence of mechatronic technology are the following [16], [17]:

- the development of artificial intelligence using information technology, leading to the formation of a global information infrastructure of the world economy and the world community;

- exploration of outer space, the bottom of the seas and oceans, including the polar circles of the planet, ultra-deep underground workings and the use of these spaces for industrial purposes;

- the creation and industrial use of high-temperature superconductors in the systems of production and distribution of electricity, as well as in all mechatronic systems;

- development of computer-aided design and production of mechatronic components and a significant expansion of the use of robotics [3].

The development of the technosphere is accompanied by experiment and modeling [9], [12], while both experiment and modeling are based on the analysis of trends in the development of energy, engineering and "green" construction standards, which determined the appearance of the next dominant technical and technological wave. In a series of successive waves of approximately dominating technologies using structural materials and energy carriers at intervals of approximately 50-70 years: solid fuel in a steam engine, liquid fuel in an internal combustion engine, electricity in an electric motor, nuclear and thermonuclear energy sources, electrochemical accumulators and electrostatic converters in mechatronic devices. With the same periodicity, the levels of experimentation and modeling of the structural and technological complexity of devices, characterized by the number of interacting components, changed. The level of experiments and modeling varied and increased. Initially, mechanical automatic machines appeared, then electromechanical 
automatic machines and robots, then electronic devices and computers and, finally, mechatronic devices and mechatronic technologies. Artificial intelligence is characteristic of mechatronic objects at all stages of the life cycle.

Mechatronics, as a concept for the future direction of development of production technology and equipment of new generations, was developed and originated in the 60-90s of the twentieth century on the wave of globalization of information and intellectual development potentials [2], [5].

The integration of the disciplines forming these mechatronics and the indicated development potentials leads to the emergence of macromechatronic and micromechatronic components of the devices, nanomechatronic and subnano mechatronic structures and media based on molecular and atomic decomposition and synthesis of materials using the energy of weak interactions of the microcosm [5].

\section{Bifurcation of the Mechatronic Technosphere}

Mechatronic technosphere is a set of systems of technological robots together with the whole set of technical means, all components of which are not directly related to the anthropometric parameters of man, are reduced to microscopic size with the acquisition of properties characteristic of all biological systems in the biosphere, which include autonomous power supply, self-reproduction, self-organization and self-improvement [2], $[5]$.

The mechatronic technosphere of the XX1st century will become "green", acquiring nature-like qualities, and will reach a new level of development, acquiring, in a certain sense, the properties of the biosphere - self-development and self-improvement. Human reason and will subordinate the development process of the "green" technosphere to the interests of nature and, accordingly, people. However, the biodiversity restoration resources of nature are limited and, therefore, the main criterion set by man based on the goal of selfpreservation in the XXI century will be the criterion of the ecological balance of the "green" mechatronic technosphere and biosphere, which should reverse the detrimental process of reducing the number of biological species the planet.

Experimentation and modeling of energy facilities can lead to instability in their location in an ecological sense.

Today in the theory and practice of management there is a high interest in the ideas of self-organization of systems and organizational structures. How to generate self-organizing structures in formal organizations conducting experimental research and research for energy construction is a central issue. Experimentation and modeling of energy facilities can lead to instability in their location in an ecological sense.

An analysis of the development priorities of the energy sector of the economy from the standpoint of the paradigm of organization and innovation sustainability based on environmental management principles makes it possible to develop an approach to building a model for managing the environmental safety of a power facility.

The permissible reliability of the study is provided by well-known tools: mathematical models of optimization and the mathematical apparatus of motion stability according to the classical theory of automatic control with the application of a statistical probabilistic approach to the environmental parameters of the energy facility.

\section{Innovation Sustainability Model}

We interpret the innovation process on the territory of the energy facility by increasing the number of new structural components in the structure of the facility, new waste and adding 
the total energy saturation of the facility [10], [11]. A fragment of the information space in conditional coordinates - waste $\mathrm{M}(\mathrm{Kg})$, components $\mathrm{N}$ (pieces), energy $\mathrm{E}(\mathrm{kW})$ to build the trajectory of innovative development of the power engineering object can be illustrated graphically in Figure [2], [5].

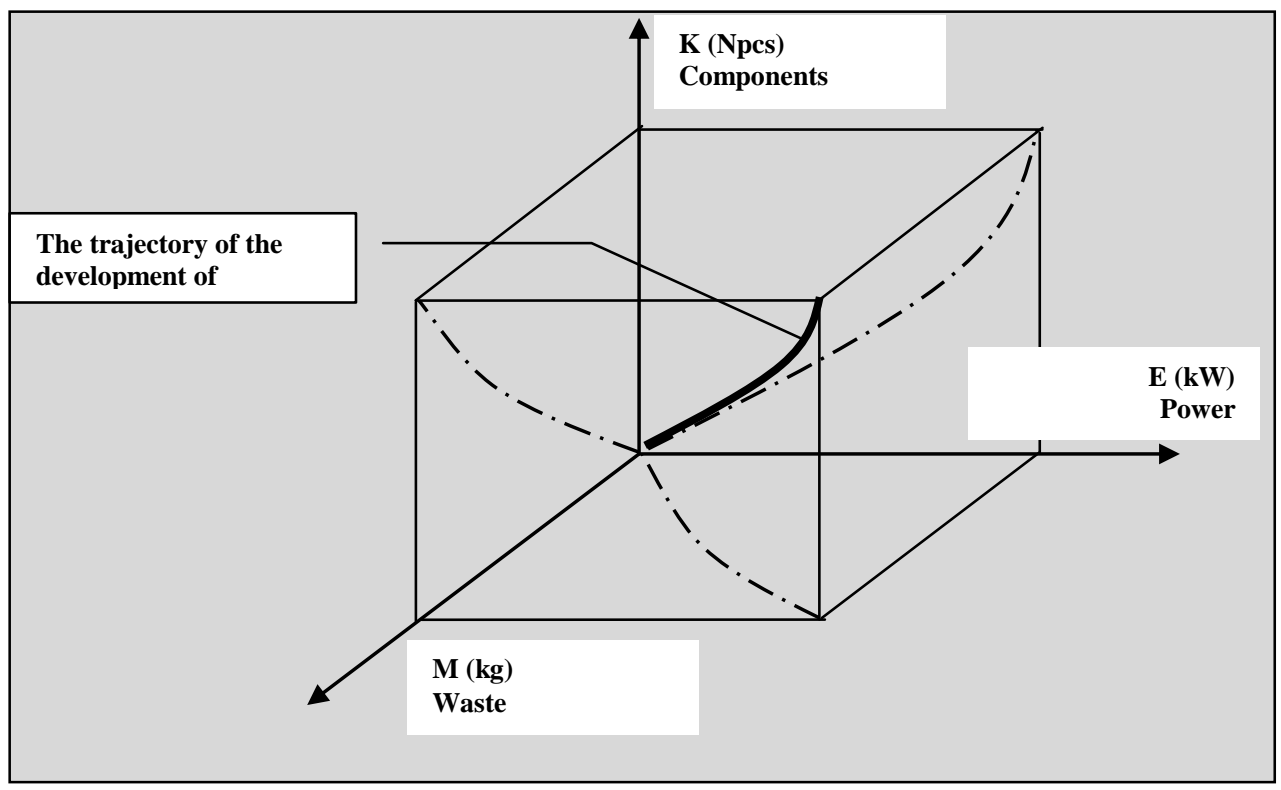

Fig. 1. Graphic interpretation of the trajectory of changing the position of point $X$ in the information coordinates of $\mathrm{K}$ - components; $\mathrm{E}$ is energy; $\mathrm{M}$ - waste.

In the general case, the equation of the trajectory of "displacement" of the parameters of the territory in the information environment of the environment can be represented as [1]:

$$
a_{0} \frac{d^{n} X}{d t^{n}}+a_{1} \frac{d^{n-1} X}{d t^{n-1}}+\cdots+a_{n-1} \frac{d X}{d t}+a_{n} X=0
$$

or in symbolic form

$$
\left(a_{0} p^{n}\left|a_{l} p^{n \sqcup l}\right| \quad \ldots\left|a_{n \sqcup 1}\right| a_{n}\right) X \mid O
$$

where $a_{0}, a_{1}, \ldots a_{n}$ are the coefficients of the equation of the development trajectory, depending on the parameters of the territorial innovation system, and $\mathrm{X}$ is the relative deviation of the controlled value of the environmental parameter in the territory. Since analyzing the innovation sustainability of the system, the free development of the territory is considered, the initial conditions for moving the position point of the energy facility in the environmental parameters along the development trajectory can be represented in the most general case as follows [1]:

$$
X(0) । \quad X_{0}, \quad X(0)^{(i)} \mid X_{0}^{(i)}(i|1,2,3, \ldots n| 1),
$$

where (i) is the order of the derivative. In this case, the solution of the homogeneous equation

$$
a_{0} \frac{d^{n} X}{d t^{n}}+a_{1} \frac{d^{n-1} X}{d t^{n-1}}+\cdots a_{n} X=0,
$$

equations describing the movement of the system in the free development of the territory, is determined by the expression: 


$$
X(t)=C_{1} e^{p_{1} t}+C_{2} e^{p_{2} t}+\cdots+C_{n} e^{p_{n} t}=\sum_{k=1}^{n} C_{k} e^{p_{k} t},
$$

where $p_{1}, p_{2}, \ldots p_{n}$ are the roots of the characteristic equation

$$
a_{0} p^{n}\left|a_{l} p^{n \sqcup 1}\right| \ldots\left|\quad a_{n \sqcup l} p\right| a_{n} \mid \quad 0
$$

and $C 1, C 2, \ldots C n$ are constants determined by the initial conditions.

In order for the innovation system in the territory to be sustainable, this solution must satisfy the requirement

$$
\lim _{t \rightarrow \infty} X(t)=0
$$

Obviously, this is possible when

$$
\sum_{k=1}^{n} C_{k} e^{p_{k} t} \rightarrow 0
$$

Let us find the restrictions imposed on the roots $p_{l}, p_{2}, \ldots p_{n}$, under which the territory is innovatively sustainable. We distinguish among the roots of $\rho$ separately real $\alpha_{0}$ and complex $\alpha_{k} \pm j \omega k$. Here the indices $\mathrm{n}$ and $\mathrm{k}$ can take different integer values. Then the solution $X(t)$ can be written in this form [1]:

$$
X(t)=\sum_{v=1}^{S} C_{\nu} e^{\alpha_{v} t}+\sum_{k=1}^{n-S} C_{k} e^{\alpha_{v} t} \sin \left(\omega_{k} t+\Psi_{k}\right)
$$

( $S$ - the number of real roots) Suppose first that all the roots of $\mathrm{p}$ are simple.

Obviously

$$
\lim _{t \rightarrow \infty} X(t)=0
$$

if each of the components of the right-hand side tends to zero. The latter will take place when $\alpha$ $<0$, that is, the real parts of all roots of the characteristic equation will be negative. If a real or complex root has multiplicity $r$, then among the components of the solution $X(t)$ the terms will be members of the form:

$$
\left.C_{1}^{\prime}+C_{2}^{\prime} t+\cdots+C_{r}^{\prime} t^{r-1}\right) e^{\alpha_{v} t}
$$

and

$$
\left(C_{1}^{\prime \prime}+C_{2}^{\prime \prime} t+\cdots C_{r}^{\prime \prime} t^{r-1}\right) \cdot e^{\alpha_{k} t} \sin \left(\omega_{k} t+\Psi_{k}\right)
$$

For negative real parts of the roots of the characteristic equation $(\alpha<0)$, the presence of factors of polynomials from $t$ will not affect the final result, because $e^{\alpha t}$ decreases as $t \rightarrow \infty$ faster than the polynomial from $t$ grows

$$
\lim _{t \rightarrow \infty}=\left(C_{1}^{\prime}+C_{2}^{\prime} t+\cdots+C_{r}^{\prime} t^{r-1}\right) e^{\alpha_{v} t}=0
$$

We next consider the case of zero and pure imaginary roots, that is, when $\alpha$ has $\alpha|| \mid 0$. Here the question of innovation sustainability is solved in two ways. With a simple zero root in the general solution there will be a constant component, which will determine the steady-state error. If the root is zero and multiple, then the system will be unstable, since in the solution there will be a term of the form: $C_{0}|| C_{l} t|\ldots| \mid C_{2} t^{r \sqcup l}$, which increases unlimitedly as $t \rightarrow \infty$ 


\section{Results and conclusions}

The model of the stability of the motion of a mechanical system in the classical form of a mathematical solution does not give a reliable result of determining the bifurcation points of a random process. The probabilistic nature of the process of changes in the environment under the influence of innovations in power engineering objects requires using a different model, for example, a soft measurement model, or upgrading the proposed deterministic model and continuing research.

\section{References}

1. M. Slesarev, E. Pankratov, V. Fedorov, MATEC Web Conf., 86, 01022 (2016)

2. M. Slesarev. Abstract of dissertation for the degree of Doctor of Technical Sciences. 43. (Moscow, NRU MGSU - 2007)

3. M. Slesarev IOP Conf. Ser.: Mater. Sci. Eng. 365022055 (2018)

4. M. Slesarev. IOP Conf. Ser.: Mater. Sci. Eng. 365022038 (2018)

5. M. Slesarev, Mash. Ents Production technology machines. Volume III-8. Technologies, equipment and control systems in electronic engineering. Part III. 714731 (2000)

6. M. Slesarev, N. Dap MATEC Web of Conferences 196, 04022 (2018)

7. M. Slesarev, V. Telichenko, N. Dap MATEC Web of Conferences 251, 02010 (2018)

8. M. Slesarev, V. Telichenko IOP Conf. Ser.: Mater. Sci. Eng. 456012126 (2018)

9. V. Telichenko, M. Slesarev, Vestnik MGSU, 13, 5, 558-567 (2018)

10. M. Slesarev, Mechatron. Mech. Automat. Electron. Informat.1, 11-16 (2000)

11. M. Slesarev. Mechatron. Mech. Automat. Electron. Informat.2, 12-14 (2000)

12. I.Engovatov, L. Alimov, M.Slesarev MATEC Web of Conferences. 8604044 (2016)

13. V. Telichenko, M. Slesarev, Constr. Mater. Equip. Technol. 21 st Cent. 2 6-7(1999)

14. V. Telichenko, M. Slesarev, X11 Polish-Russian Sem. on Foundation of Civil Eng. Proc Moscow-Nizhny Novgorod-Warsaw 445-452 (2003)

15. G. Makarov, M. Slesarev. Ecolog. Syst. Devic. 2 39-45 (2006)

16. V. Telichenko, M. Slesarev, Proc. 1X Polish-Russian Sem. Theoretical Bases of Construction (Moscow: Association Civi Engineering Higher Education Press) 251260 (2000)

17. V. Telichenko, V. Gutenev, M. Slesarev, J. Ecol. Urban. Terr. 6-11 (2006) 